Sweden, July 4th to 19 th, 1968. This declaration was approved and recommended by the Assembly in its essential features. Compared with the Vatican Council's Decree on the Instruments of Social Communication ("Inter mirifica") it shows some correspondence in principal, emphasizing, however, different conditions of communication activities in various countries. The general attitude of the declaration is best characterized by self-criticism, when in fact the decree is stressing church authority in communication. Both documents take notice of the missionary feasibilities in communication. In contrast to the Roman Catholic decree the Uppsala declaration states the responsibility of the churches to provide free flow of communication in admitting free access to news. There is hope for ecumenical dialogue on problems of communication since many questions were left unanswered in both papers.

\title{
RESUMEN
}

A la 4. Reunión General del Consejo Ecuménico de Iglesias, celebrado en Uppsala del 4 al 19 de Julio de 1968, le fue presentado, entre otros una Declaración sobre la „Iglesia y los Medios de Comunicación Social". El contenido esencial de esta Declaración fue aceptado y recomendado por la Reunión General. Si comparamos esta Declaración con el Decreto Conciliar sobre los Medios de Comunicación Social („Inter mirifica"), descubriremos una concordancia en los rasgos fundamentales. No obstante, la Declaración de Uppsala se ocupa sobre todo de las diversas condiciones de la actividad periodîstica en los diferentes paîses. Su postura es autocrîtica, mientras que el Decreto Conciliar acentúa fuertemente la primacîa de la Iglesia frente al periodismo. Ambos documentos constatan la importancia misional de las actividades periodistîcas. En oposición al punto de vista católico romano, la Declaración de Uppsala acentúa expresamente la obligación de las Iglesias de dar a conocer libremente sus informaciones. Como en ambas declaraciones han quedado muchos problemas sin resolver, abiertos, existen fundadas esperanzas de que se abra un diálogo ecuménico sobre los problemas de Comunicación.

\section{Presse und Funk im Territorium von Papua und Neuguinea (II)}

\author{
von Franz-Josef Eilers
}

\begin{abstract}
Dem ersten Teil dieses Beitrags in Nr. 3 CS (S. 197-208) über die Presse im Territorium von Papua und Neuguinea folgen eine tabellarische Ubersicht der Zeitungen und Zeitschriften und eine Darstellung der Situation des Hörfunks.
\end{abstract}

\section{Ubersicht über Zeitungen und Zeitschriften in Papua und Neuguinea}

In der nachfolgenden Ubersicht handelt es sich um den ersten Versuch einer Gesamtschau der Presse im Territorium Papua und Neuguinea. Die einzelnen Angaben beruhen auf eigenen Untersuchungen, die ergänzt wurden durch Angaben in den 
offiziellen Jahresberichten über Papua und Neuguinea vom Department of Territories in Canberra und in der "Annotated Bibliography of Select Government Publications on Australian Territories 1951-1964" vom gleichen Department (Canberra 1965).

\begin{tabular}{|c|c|c|c|}
\hline Titel & Erscheinungsort & Auflage & Bemerkungen \\
\hline \multicolumn{4}{|l|}{ 1. Zeitungen } \\
\hline South Pacific Post & Port Moresby & 5000 & $\begin{array}{l}\text { gegr. } 1950,3 \times \text { wöchentlich, } \\
\text { englisch }\end{array}$ \\
\hline $\begin{array}{l}\text { The New Guinea Times- } \\
\text { Courier }\end{array}$ & Lae & 3500 & $\begin{array}{l}\text { aus } 21957 / 58 \text { gegr. Blättern ent- } \\
\text { standen, } 2 \times \text { wöchentlich } \\
\text { Druckort: Port Moresby, engl. }\end{array}$ \\
\hline Nu Gini Toktok & Port Moresby & 3000 & $\begin{array}{l}\text { gegr. } 1962,1 \times \text { wöchentlich, } \\
\text { Pidgin-Englisch }\end{array}$ \\
\hline Government Gazettes & Port Moresby & - & $\begin{array}{l}\text { gegr. } 1884 \text { als: "British New } \\
\text { Guinea“, ab 1906: "Papua“, ab } \\
\text { 1945: "Papua-New Guinea “, ab } \\
\text { Juni 1949: Government Gazettes. }\end{array}$ \\
\hline \multirow[t]{2}{*}{$\begin{array}{l}\text { Our News } \\
\text { Nius bilong yumi } \\
\text { Iseda Sivarai }\end{array}$} & $\begin{array}{l}\text { Port Moresby } \\
\text { Port Moresby } \\
\text { Port Moresby }\end{array}$ & $\begin{array}{l}6000 \\
4000 \\
1300\end{array}$ & $\left.\begin{array}{l}\text { englisch } \\
\text { Pidgin-Engl. } \\
\text { Police Motu }\end{array}\right\} \begin{array}{l}\text { gegr. ca. 1962, } \\
\text { gleicher Inhalt } \\
\text { bei allen drei } \\
\text { Blättern; }\end{array}$ \\
\hline & & & $\begin{array}{l}14 \text { täg., Hrsg.: Department of } \\
\text { Information and Extension } \\
\text { Services }\end{array}$ \\
\hline United Nations Newsletter & Port Moresby & 500 & $\begin{array}{l}\text { wödhentlich, Hrsg.: Informations- } \\
\text { zentrum der UNO, Pidgin-E. und } \\
\text { Motu (je } 300 \text { u. } 200 \text { Exempl.) }\end{array}$ \\
\hline
\end{tabular}

2. Zeitschriften

2) Zeitschriften amtlicher Stellen

aa) Zentrale Regierungsstellen des Territoriums (alphabetisch nach herausgebenden Abteilungen). Anmerkung: Nicht aufgeführt sind in diesem Verzeichnis die vierteljährlich oder jährlich erscheinenden Blätter des Büros für Statistik mit statistischen Angaben zu verschiedenen Bereichen des öffentlichen Lebens. Vgl. dazu: Annotated Bibliography of Select Government Publications on Australian Territories 1951-1964, Department of Territories, Canberra, 1965, $41 \mathrm{f}$.

The Apprentice Port Moresby

The Papua and New Guinea Port Moresby Agricultural Journal

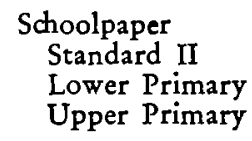

Port Moresby gegr. 1959, halbjährl., Hrsg.: Apprenticeship Board

1500 gegr. 1953, vtlj., Hrsg.: Department of Agriculture, Stock and Fisheries; vor dem Krieg $8 \mathrm{Jahr}$ gänge in Rabaul unter dem Titel: „New Guinea Agricultural Gazette"
gegr. 1963
monatlich, englisch
Hrsg.: Department of
Education 


\section{Titel}

Teachers' Paper

The Papua-New Guinea Journal of Education

Listen and Learn

Preparatory

Standard I

Broadcasts for School The Papua and New Guinez
Villager

Infant Welfare Newsletter

Papua and New Guinea Medical Journal

Industrial Review

Welfare Quarterly

bb) lokale Regierungsstellen und Councils

$\begin{array}{ll}\text { Bola Council News } & \begin{array}{l}\text { Bola, } \\ \text { Bew Britain } \\ \text { Buin }\end{array} \\ \text { Challenge } & \text { Port Moresby } \\ \text { Council Gazette } & \text { Finschhafen } \\ \text { Council News } & \text { Kavieng (?) } \\ \text { Gulf District News } & \text { Kerema } \\ \text { Hari Dina } & \text { Port Moresby } \\ \text { Kamonrai } & \text { Baluan } \\ \text { Sepik News } & \text { Wewak } \\ \text { Siwai Rure } & \text { Boku/Buin } \\ \text { V. A. C. } & \text { Samarai }\end{array}$

Wewak News

Erscheinungsort Auflage Bemerkungen

Port Moresby

Port Moresby $\quad 4000$

Port Moresby

Port Moresby

Port Moresby

Port Moresby

Port Moresby

Port Moresby

Port Moresby

Port Moresby gegr. 1963, unregelmäßig

Hrsg.: Department of Education, Englisch;

1500 gegr. 1963; $3 \times$ jährl., Department of Education, Engl., früher: South Pacific Journal of Education

jährlich. Hrsg.: Department of Education in Verbindung mit "Australian Broadcasting Commission"

gegr. 1927, jetzt: Dep. of Education

- gegr. 1958, monatlich, Department of Public Health

- gegr. 1955, Dep. of Public Health

- gegr. 1963, viertelj., Department of Labour

800 vervielf., Dep. of Native Affairs
- Hrsg.: Bola Local Government Council, Talesea Subdistr.

- Hrsg.: Sub-District Office Buin, Bougainville Distr.

- Hrsg.: Administrative College, Port Moresby

- monatl., Hrsg.: Yabim-Kotte Council, Morobe District, Pidgin-Englisch

- monatl., Hrsg.: Tikana-Council, New Ireland Distr., Pidgin-Engl.

500 monatl., Dep. of Native Affairs, Kerema, Gulf Distr., Englisch

- Hrsg.: Fairfax Local Government Council; Motu

- monatl. Hrsg.: Baluan Council, Manus Distr., Pidgin-Engl.

- zweimonatl., Hrsg.: Council Chambers, Wewak, Sepik Distr.

- Hrsg.: Siwai Local Government Council, Boku; Bougainville District

- Village Agricultural Commitees, freie Folge; Hrsg.: District Agricultural Officer, Samarai, Milne Bay District.

- viertelj., Hrsg.: Department of Native Affairs, Wewak 
b) Von christlichen Missionen herausgegebene Zeitschriften

Catholic News

The Challenge

Church News

Compass

The Contact

Harim

Idupa

The Kairiru

Katolik Nius

Kombobro

The New Guinea Lutheran Madang

St. Michael's Messenger

Methodist Messenger
Sohano

Menyamya (Lae)

Rabaul

Rabaul

Port Moresby

Port Moresby

Wabag

Kairiru Is.

Vunapope

Dogura

Kieta

?
- gegr. 1959, monatl., Cath. Mission (Marist Mission: Marcellin Press) Tsiroge, Bougainville Distr., Englisch und teilw. Pidgin, seit 1. 1. 1967 eingestellt.

5000 jährl., Hrsg.: Australian Lutheran Mission, Menyamya, via Lae; Engl., gedruckt in Australien, Offset

600 gegr. 1957, monatl., Hrsg.: Parish of St. George the Martyr, Rabaul and District, Engl. (Anglikaner)

350 vierteljährl., Hrsg.: Seventh Day Adventists Mission, Box 391, Rabaul; Engl., vervielf.

500 monatl., Hrsg.: Salvation Army, Box 201, Port Moresby, Engl., Offset

1400 zweimonatl., Hrsg.: Missionary Association of Papua and New Guinea (MAPANG), Box 29, Port Moresby. Druck: Stanmore Missionary Press, Sydney, farb. Offset, Pidgin, vgl. auch "Onward“

4000 monatl., Hrsg.: Lutheran Mission, Missouri Synod, Wabag vervielf., Enga-Sprache und 1 Seite PidginEnglisch

120 freie Folge; Schülerzeitschrift der St. Xavier's Junior High School, Marist Brothers, Cath. Mission, Kairiru Is., Sepik Distr. vervielf., Englisch

3700 gegr. 1937, monatl., Cath. Mission Vunapope, New Britain; in ganz Neuguinea (außer Papua) verbreitet; Pidgin-Engl.

500 zweimonatl., Lehrerzeitschrift, Hrsg.: New Guinea Anglican Mission, Dogura, Milne Bay Distr., Englisch, vervielf.

? monatlich, Hrsg.: Evangelical Lutheran Church of New Guinea; Druck: Lutheran Mission Press, Nagada b. Madang. Ausgaben in Englisch und Pidgin-Englisch. Weitere Ausgaben in 2 Lokalsprachen (1964 in Vorbereitung)

- Hrsg.: Cath. Mission Tubiana, Kieta, Bougainville Distr.

- Hrsg.: Methodist Overseas Mission, Englisch 


Titel Erscheinungsort Auflage Bemerkungen

Milne Bay Monthly

A Nilai ra dovot

Onward

Programme Guide

Talaigu (Mein Freund)

Tapwardro Teterina

Wewak

Yule Island News
Daio (Samarai)

Rabaul

Port Moresby

Kambubu

Vunapope

East Cape

Wewak

Yule Is.

\section{-}

sion Daio, via Samarai, Engl.

4000 gegr. 1909; monatlich, Hrsg.:

Methodist Overseas Mission

(Methodist Mission Press) Rabaul. Lokalsprache und teilweise Pidgin-Englisch

2000 zweimonatl. (bis Juni 1964 vierteljährl.), Hrsg.: Missionary Association of Papua and New Guinea (MAPANG), Box 29, Port Moresby. Druck: Stanmore Missionary Press, Sydney, Englisch, farbiger Offset-Dr., vgl. auch: „Harim"

600 vierteljährl.- Programmvorschläge für religiöse Jugendmeetings, Hrsg.: Seventh Day Adventists, Kambubu, Engl.

3400 gegr. 1926, monatlich, Cath. Mission Press, Vunapope, Lokalsprache (Tolai)

- Hrsg.: Methodist Overseas Mission, East Cape via Samarai, Milne Bay Distr.

- gegr. 1964 (vorher: WewakGlodken), Hrsg.: Cath. Mission of the Divine Word (Mgr. Leo Arkfeld), Wewak. Mitteilungsblatt für Missionsfreunde: englische und deutsche Ausgabe.

600 monatl., Hrsg.: Cath. Mission

(Comet-Press), Yule Is., Central Distr.; Englisch

c) Zeitschriften privater Herausgeber und Vereinigungen

\section{Australian Boy Scouts Association, Papua-New Guinea Branch}

Goroka Newsletter

The Journal of the Papua and New Guinea Society

Kibi

Kundiawa News

Rigo Hakala
Port Moresby

Goroka

Port Moresby

Port Moresby

Kundiawa

Rigo
- Mitteilungsblatt der gleichnamigen Organisation; Englisch

- monatlich; ?

Seit 1966/67; in Verbindung mit der Universität von Papua und Neuguinea, halbjl.

- Hrsg.: Federation of Native Associations for the Cooperative Society

- Hrsg.: Rigo Community Development Committee, Kwikiia, Rigo, Central Distr., Englisch 


\begin{tabular}{|c|c|c|c|}
\hline Titel & Erscheinungsort & Auflage & Bemerkungen \\
\hline Tolai Cocoa Project News & Rabaul & - & monatlich; ? \\
\hline Volunteer & Port Moresby & - & $\begin{array}{l}\text { Hrsg.: Papua and New Guinea } \\
\text { Volunteer Rifles, Port Moresby, } \\
\text { Englisch }\end{array}$ \\
\hline $\begin{array}{l}\text { The New Guinea Highlands } \\
\text { Bulletin }\end{array}$ & Goroka & 1200 & $\begin{array}{l}\text { gegr. 1959; vierteliährlich, } \\
\text { Hrsg.: Highland Farmers and } \\
\text { Settlers Association (Mr. Jan } \\
\text { Downs), Box 111, Goroka; } \\
\text { gedruckt in Sydney; Engl., gele- } \\
\text { gentlich Pidgin-Englisch }\end{array}$ \\
\hline $\begin{array}{l}\text { New Guinea Research Unit } \\
\text { Bulletin }\end{array}$ & Port Moresby & 1000 & $\begin{array}{l}\text { circa vierteljährlich, Wissenschaft- } \\
\text { liche Publikation in Zusammen- } \\
\text { arbeit mit der Australian National } \\
\text { University Canberra (A. N. U.), } \\
\text { Englisch }\end{array}$ \\
\hline Women's Club Newsletter & Port Moresby & 一 & $\begin{array}{l}\text { monatlich, Mitteilungsblatt der } \\
\text { gleichnamigen Organisation }\end{array}$ \\
\hline
\end{tabular}

\section{Rundfunk}

Die Geschichte des Rundfunks im Territorium Papua und Neuguinea beginnt, technisch gesehen, schon vor dem Ersten Weltkrieg, als die deutsche Reichsregierung wenige Monate vor Kriegsausbruch einen starken Postsender in Bitapaka bei Rabaul (New Britain) in Betrieb nehmen konnte. Der Sender, dessen Fundamente noch heute zu sehen sind, war vor allem dafür bestimmt, die Verbindung mit der weit entfernten deutschen Heimat aufrecht zu erhalten. ${ }^{20}$ Schon zwei Tage nach Kriegsausbruch 1914 bat die englische Regierung Australien, diesen Sender zu besetzen. Am 12. September 1914 konnten dann australische Truppen in die Anlagen eindringen und sie beschlagnahmen. ${ }^{21}$

\section{A. Sender der Australian Broadcasting Commission (ABC)}

Nach der Besetzung von Papua und Neuguinea durch die Japaner im Zweiten Weltkrieg wurde am 26. Februar 1944 in Port Moresby ein Militärsender errichtet. Die notdürftig in Baracken untergebrachte Station wurde 1946 von der Australian Broadcasting Commission übernommen und ging unter den bereits bis dahin gebrauchten Sendezeichen 9 PA im Mittelwellenbereich (1 $250 \mathrm{KHZ}, 240 \mathrm{~m}$ ) und VLT 6 im Kurzwellenbereich auf Sendung. Die Stärke der beiden Sender betrug jeweils 2 KW. Inzwischen konnte am 5. September 1964 für die ABC-Station in Port Moresby ein neues Sendegebäude in Betrieb genommen werden, dessen technische Einrichtung durch das zuständige Postministerium in Canberra installiert wurde. Ferner konnte 1962/63 der bestehende Kurzwellensender auf eine Stärke von $10 \mathrm{KW}$ erhöht und durch einen zweiten, gleichstarken Sender (VLK) ergänzt werden. Am 15. Dezember $1962 \mathrm{nahm}$ die erste Regionalstation der ABC in Rabaul (9 RB) auf der Mittelwelle $(810 \mathrm{KHZ}, 370 \mathrm{~m})$ mit 2 Kilowatt Sendeleistung ihren Betrieb auf.

Die Sender der ABC in Port Moresby senden ihr Programm werktäglich von 6.00 bis $24.00 \mathrm{Uhr}$ und sonntags von 6.30 bis $24.00 \mathrm{Uhr}$. Die Station in Rabaul hat 
von 6.00 Uhr bis 13.45 Uhr und von 16.00 bis 22.45 Uhr ein eigenes Programm. Rund 40 Australier und 3 einheimische Kräfte sind unmittelbar für den Sende- und Redaktionsbetrieb in Port Moresby eingesetzt. Es gibt u. a. neben der eigenen Nachrichtenabteilung mit 22 Korrespondenten im ganzen Territorium eine eigene Schulfunk-, Landfunk- und Transskriptionsabteilung. Neben den von Australien übernommenen Nachrichtendiensten verbreitet ABC Port Moresby Lokalnachrichten in einfachem Englisch, Pidgin-Englisch und Motu. Seit Sommer 1964 wird auch eine in einfachem Englisch gefaßte Sendung von Weltnachrichten ausgestrahlt, die speziell für die einheimische Bevölkerung bestimmt ist und die deswegen zu den eigentlichen Nachrichten Erklärungen und Ergänzungen enthält: zum besseren Verständnis für politisch unerfahrene Hörer.

Besonders $z u$ erwähnen ist das Bestreben der ABC, auch einheimische Kräfte für den Rundfunk heranzubilden. So stehen bereits mehrere Volontäre vor dem Abschluß ihrer Ausbildung. Eine ihrer Hauptaufgaben wird zunächst die Ansage, Formulierung und Ubersetzung von Nachrichten und Berichten für die einheimische Bevölkerung sein. Als schulische Voraussetzung für den einheimischen Nachwuchs wird als Minimum die abgeschlossene „Form 3“ $(=3$. Klasse der Secondary School, ca. Obersekunda) gefordert.

Nach dem Jahresbericht von 1963/64 haben die Stationen der ABC in Neuguinea im Berichtsjahr 24326 Sendestunden (1962/63: 15 266; 1961/62: 12730) ausgestrahlt. Eine Analyse des Programms für Neuguinea ergibt u. a. folgende Schwerpunkte:

1. Nachrichtensendungen: Es werden drei Arten von Nachrichtensendungen unterschieden:

a) Nachrichten aus Australien und Ưbersee (Australian and Overseas News). Sie werden, abgesehen von einem Dienst in einfachem Englisch mit Erklärungen, der in Port Moresby erstellt und werktags um 18.00 Uhr ausgestrahlt wird, und einem von der BBC London übernommenen Nachrichtendienst um 23.00 Uhr, - von der Nachrichtenzentrale der ABC in Melbourne übernommen. Das Zeit-Schema der Nachrichtendienste sieht folgendermaßen aus:

Australian and Overseas News:

$\begin{array}{lc}\text { werktags } & \text { sonntags } \\ 6.00-6.05 & 6.45-7.00 \\ 7.45-7.55 & 9.00-9.10 \\ 10.25-10.28 & 12.30-12.40 \\ 12.30-12.40 & 13.30-13.40 \\ 13.30-13.40 & 19.00-19.10 \\ 16.00-16.05 & 21.00-21.10 \\ 18.00-18.10 \text { (einf. Englisch) } & 22.00-22.10 \text { (BBC News) } \\ 19.00-19.10 & \\ 21.00-21.10 & \\ 23.00-23.10 \text { (BBC News) } & \end{array}$

b) Nachrichten aus dem Territorium (Territory News). Diese Sendungen werden von der Nachrichtenabteilung in Port Moresby erstellt und in englischer Sprache ausgestrahlt. Das Zeitschema dieses Dienstes sieht folgendermaßen aus:

$\begin{array}{cc}\text { werktags } & \text { sonntags } \\ 7.55-8.00 & 19.10-19.15 \\ 19.10-19.15 & 21.10-21.15 \\ 21.10-21.15 & 22.10-22.15 \\ 23.10-23.15 & \end{array}$


c) Nachrichten aus Papua und Neuguinea (Papua and New Guinea News).

Dieser nur werktäglich gesendete Dienst enthält jeweils fünf Minuten Lokalnachrichten in einfachem Englisch, Pidgin-Englisch und Police-Motu. Das Zeitschema dieser Dienste sieht folgende Sendungen vor:

$$
\begin{aligned}
& \text { werktags } \quad 6.05-6.20 \\
& 20.00-20.15
\end{aligned}
$$

2. Schulfunk: Die Schulfunksendungen von $A B C$ erfreuen sich im ganzen Territorium großer Beliebtheit. Durch die z. T. vom Department of Education in Port Moresby zur Verfügung gestellten Rundfunkempfänger konnten sich im Berichtsjahr 1963/64 bereits $32 \%$ aller Schulen an den Sendungen beteiligen. Die Sendezeiten für den Schulfunk liegen von montags bis freitags vormittags zwischen 8.30 Uhr und 9.30 $\mathrm{Uhr}$ und zwischen 11.00 Uhr (bzw. 11.25 Uhr) und 12.00 Uhr. Die Sendeeinheit für einzelne Klassen bzw. Themenbereiche beträgt jeweils 15 Minuten.

3. Landfunk: Der Landfunk meldet sich bei ABC Port Moresby regelmäßig in der Zeit von $12.40 \mathrm{Uhr}$ bis $13.00 \mathrm{Uhr}$. Außerdem gibt es täglich Wetterinformationen, Meldungen zu Kakao-, Kopra- und Kaffeepreisen, zum Schiffs- und Flugverkehr. An einzelnen Tagen der Woche sind außerdem zusätzliche Sendungen zu hören (z. B. dienstags: $20.30 \mathrm{Uhr}$ bis 20.45 Uhr, Planters Corner; freitags: 13.00 Uhr bis 13.15 Uhr, Farm and Home; mittwochs: 18.10 Uhr bis 18.30 Uhr die Sendung des Landwirtschaftsbeamten der Regierung für die einheimische Bevölkerung: Toktok bilong Didiman).

4. Frauenfunk, Kinderfunk, Musik: Der Frauenfunk meldet sich werktäglich von $9.30 \mathrm{Uhr}$ bis 10.10 Uhr zur "Women's Session" und um 14.40 Uhr heißt es "Mainly for Women " (bis $15.00 \mathrm{Uhr}$ ). Montags bis freitags ist von $16.05 \mathrm{Uhr}$ bis $17.00 \mathrm{Uhr}$, samstags von 17.30 Uhr bis 18.00 Uhr und sonntags von 16.45 Uhr bis $17.15 \mathrm{Uhr}$ die „Children's Session ${ }^{\alpha}$. Der Musikliebhaber (For the musiclover) findet u. a. täglich, außer samstags und sonntags ein Programm von $15.00 \mathrm{Uhr}$ bis $16.00 \mathrm{Uhr}$, das meist klassische oder halbklassische Musik enthält. Daneben sind etwa zweimal wöchentlich größere Konzerte im Abendprogramm, und täglich gibt es wenigstens von $20.15 \mathrm{Uhr}$ bis $20.30 \mathrm{Uhr}$ (meist aber auch noch zu weiteren Zeiten) für den Hörer die Gelegenheit, zum Wunschkonzert seine Wünsche zu äußern.

\section{B. Rundfunkstationen der Verwaltung}

Verschiedene kommerzielle Gruppen und christliche Missionen versuchten nach dem Kriege ohne Erfolg, bei der australischen Regierung und der Verwaltung des Territoriums eine Lizenz zur Errichtung eigener Sender zu bekommen. Das Bemühen dieser Gruppen wurde von Jahr zu Jahr stärker, zumal die ABC-Stationen nur einen geringen Einfluß auf die einheimische Bevölkerung gewannen. $1961 \mathrm{kam}$ es dann unter dem Vorsitz des Direktors für das Post- und Telegraphenwesen in Papua/Neuguinea zur Gründung eines Broadcasting Services Committee, das in seinem Schlußbericht die Notwendigkeit von eigenen Rundfunkstationen für die einheimische Bevölkerung betonte. Zugleich pflichtete man aber auch der Feststellung des damaligen Vorsitzenden der Australian Broadcasting Commission, Sir Richard Boyer, bei, daß „die vorgesehenen regionalen Sender nicht in den Aufgabenbereich der ABC fielen".22

So begann nun die Verwaltung des Territoriums, diese Regionalsender zu errichten, die redaktionell vom Department of Information and Extension Services betreut werden. Da diese Stationen ausdrücklich für die einheimische Bevölkerung errichtet sind, senden sie fast nur in einheimischen Sprachen bzw. in Pidgin-Englisch oder Police Motu. Die Errichtung der ersten Stationen in Rabaul (1961) und Wewak 
(1963) war ein voller Erfolg. Inzwischen wurden noch Sender in Kerema (1964), Goroka (1965), Daru (1965) Mt. Hagen (1966), Kieta (Bougainville 1967) und Samarai (Milne Bay 1967) eröffnet. Weitere solcher Lokalsender sind bis 1969 für folgende Orte vorgesehen: Madang, Vanimo, Lae, Popondetta, Mendi, Kavieng, Kainantu, Talasea und Wabag. Durch eine Beschränkung der Sendezeit auf die Spätnachmittagsstunden und Abendstunden können diese Lokalsender zunächst die Post- und Telegraphensender für ihre Programme benutzen (250 Watt). Radio Wewak hat inzwischen seit Anfang 1965 einen eigenen 10 Kilowatt-Sender in Betrieb genommen. Die gleiche Entwicklung und damit eine Ausdehnung der Sendezeit war 1965 für Rabaul geplant. Für sämtliche technischen Einrichtungen ist auch bei diesen Sendern (wie bei $\mathrm{ABC}$ ) das Postministerium verantwortlich.

Allen bisher errichteten Sendern des Department of Information and Extension Services ist gemeinsam, daß sie unter europäischer Leitung weithin nur mit einheimischen Kräften arbeiten. So arbeiten unter der Leitung von Jim Leigh und Jan Smeeton bei Radio Rabaul etwa 12 einheimische Kräfte als Ansager, Reporter, Redakteure usw. Ahnlich ist es bei Radio Wewak. Ein eigenes Schulungszentrum für diese einheimischen Kräfte besteht bei Port Moresby. Besonders auffallend ist der äußerst starke Hörerkontakt dieser Rundfunkstationen. So erhält Radio Wewak monatlich rund 500 Hörerbriefe, die Nachrichten und andere Mitteilungen enthalten; die Zahl der Musikwünsche stieg von 2000 in den ersten Monaten 1964 auf 5000 im Juni des gleichen Jahres. Radio Rabaul registrierte September 196412600 Musikwünsche. Gründe für diesen auffallend starken Hörerkontakt liegen nicht nur in der Neuartigkeit des Mediums Rundfunk für die bisher fast ohne moderne Kommunikationsmittel lebenden einheimischen Menschen. Sie liegen auch in dem ausgiebigen oder fast ausschließlichen Gebrauch einheimischer Sprachen oder weitverbreiteter volkstümlicher Verkehrssprachen (Pidgin, Motu). Hinzu kommt eine ausgezeichnete, auf die Interessen der Hörer abgestimmte Programmgestaltung.

So brachte Radio Wewak 1964 fast täglich wenigstens eine Stunde Wunschkonzert (bei sechsstündigem Gesamtprogramm!); in den von Radio Wewak redigierten lokalen Nachrichtendiensten werden häufig die Namen der einheimischen Nachrichteneinsender genannt. Die Beantwortung von Leserbriefen (im Funk und persönlich) wird besonders gepflegt. Ferner enthält das Musikprogramm auch einen Großteil einheimischer Musik, aufgenommen bei Festlichkeiten an verschiedenen Orten des Sendegebietes selbst oder benachbarter Gebiete. Radio Rabaul hat mit besonderem Erfolg Originalübertragungen von Council-Sitzungen und anderen öffentlichen Ereignissen ungekürzt ausgestrahlt (bis zu fünf Stunden!). Diese Ubertragungen wurden entweder live oder als Bandaufnahme gesendet. Radio Wewak geht einmal wöchentlich in das Boram Hospital in Wewak und interviewt Kranke, die auch Nachrichten zu ihren Dörfern geben können. In der halbstündigen sonntäglichen Sendung von Radio Wewak Austin Sapias plays new records könnte man den Ansatz für die Popularisierung eines Ansagers sehen. Die in Radio Rabaul entwickelte Reihe Pre-School of the Air (auch von Radio Wewak iibernommen) ist eine beliebte Sendung, die Hinweise zur Erhaltung der Volksgesundheit enthält. Zugleich wird in dieser Sendung aber auch der in Pidgin-Englisch gesprochene Satz in englischer Sprache wiederholt, und so dient diese Sendung auch der sprachlichen Weiterbildung der Hörer.

Die täglichen Sendezeiten betrugen 1964/65 für Radio Rabaul 61/2 Stunden (17.00 Uhr bis 22.30 Uhr) und für Radio Wewak 6 Stunden (17.30 Uhr bis $22.30 \mathrm{Uhr}$ ). Ahnlich dürfte es auch bei den anderen Lokalsendern $\operatorname{sein}^{23}$. 
Das Schema der Nachrichtensendungen bei Radio Rabaul sah im Sommer 1964 für die Werktage folgendermaßen aus:

18.00-18.10 ABC Weltnachrichten in einfachem Englisch

18.10-18.15 Nachrichten in der Tolai-Sprache

19.00-19.15 Lokalnachrichten in Pidgin-Englisch

20.00-20.15 ABC Territory Nachrichten in Englisch, Pidgin, Tolai

20.30-20.40 Nachrichten in Tolai

21.30--21.40 Lokalnachrichten in Pidgin-Englisch

22.00-22.10 Nachrichten in Pidgin-Englisch

In einem Prospekt des Senders Rabaul werden außerdem als Sendezeiten für einheimische Musik ausgewiesen: montags 40 Minuten, dienstags 20 Minuten, mittwochs 30 Minuten, donnerstags 25 Minuten, freitags 20 Minuten, samstags 75 Minuten und sonntags 45 Als Beispiel für ein Programmschema sei hier der Sendeplan von Radio Wewak für Dienstag und Mittwoch (Stand: Sommer 1964) wiedergegeben:

dienstags:

17.30 Uhr Opening Announcement, Program Details, "Pre-School of the Air", Popular Music

18.00 Uhr World News (ABC, Port Moresby)

18.10 Uhr Listeners' Requests

18.25 Uhr News in Pidgin

18.30 Uhr Cowboy Songs

18.45 Uhr Overseas and Coastal shipping, aircraft movements, Special announcements

18.53 Uhr Local news

19.00 Uhr Overseas and Australian-, Territory News

19.15 Uhr Popular Music

19.30 Uhr News in Pidgin

19.35 Uhr Music i kam long South Pacific

19.45 Uhr Listeners' Requests

20.00 Uhr News in English and Pidgin

20.10 Uhr Singsing bilong ol ples (einheim. Musik) Popular Music

20.35 Uhr Listeners' Requests

21.00 Uhr News in Pidgin

21.05 Uhr Music with Damian Pahpat

21.15 Uhr Listeners' Requests

21.30 Uhr Music i kam long South Pacific

21.45 Uhr Singsing bilong ol ples (einheim. Musik) Popular Music

22.00 Uhr News in Pidgin

22.05 Uhr New records

22.20 Uhr Music

22.29 Uhr Closing announcement, National Anthem

22.30 Uhr Close mittwochs:

17.30 Uhr Opening Announcement, Program Details

17.33 Uhr Children's Songs

17.45 Uhr Singsing bilong ol ples Popular Music

18.00 Uhr World News (ABC, Port Moresby)

18.10 Uhr Listeners' Requests

18.25 Uhr News in Pidgin

18.30 Uhr Toktok bilong Didiman (Landfunk)

18.45 Uhr Overseas, Coastal Shipping, aircraft movements, Special announcements, music

18.53 Uhr Local News Music

19.00 Uhr Overseas, Australian and Territory News

19.15 Uhr Popular Music

19.30 Uhr News in Pidgin

Music i kam long South Pacific

19.45 Uhr Listeners' Requests

20.00 Uhr News in English and Pidgin (ABC)

20.10 Uhr Radio Wewak Hit Parade

20.40 Uhr Listeners' Requests

21.00 Uhr News in Pidgin

21.05 Uhr Singsing bilong ol ples (einheim. Musik)

21.15 Uhr Listeners Requests

21.30 Uhr Music i kam long South Pacific

21.45 Uhr Popular Music

22.00 Uhr News in Pidgin

22.05 Uhr New records

22.20 Uhr Popular Music

22.29 Uhr Closing announcement, National Anthem

22.30 Uhr Close 
Die bis zum Jahre 1966 tätigen Lokalsender des Departement of Information and Extension Services in Papua und Neuguinea haben folgende Standorte, Wellenlängen, Sendezeichen und Sendestärke:

$\begin{array}{llrll}\text { Radio Rabaul } & (1961) & 3385 \mathrm{Kc} & \text { VL9BR } & 10 \mathrm{KW} \\ \text { Radio Wewak } & (1963) & 335 \mathrm{Kc} & \text { VL9CD } & 10 \mathrm{KW} \\ \text { Radio Kerema } & (1964) & 3245 \mathrm{Kc} & \text { VL8BK } & 250 \mathrm{~W} \\ \text { Radio Daru } & (1965) & 3304 \mathrm{Kc} & \text { VL8BD } & 10 \mathrm{KW} \\ \text { Radio Goroka } & (1965) & 2410 \mathrm{Kc} & \text { VL9CG } & 250 \mathrm{~W} \\ \text { Radio Mt. Hagen } & (1966) & 2450 \mathrm{Kc} & \text { VL9CH } & 250 \mathrm{~W}\end{array}$

\section{Die Zukunft des Funks}

Die Zahl der in Neuguinea befindlichen Empfangsgeräte für Rundfunksendungen ist nicht festzustellen. Es gibt keine Rundfunkgebühr und keine Registrationspflicht für Rundfunkgeräte. Eines läßt sich allerdings für jene Gebiete sagen, in denen bereits Sender des Department of Information and Extension Services arbeiten: Die Zahl der von den Einheimischen erworbenen Transistor-Rundfunkempfänger wächst rapide. Es dürfte bald kein Dorf mehr geben, in dem nicht wenigstens ein Rundfunkgerät vorhanden und deswegen auch ein Gemeinschaftsempfang möglich ist. Neben den Schulen erhalten von der Regierung auch Hospitäler nach und nach kostenlos Rundfunkempfänger, während die Geräte in den Dörfern meist von den Bewohnern selbst erworben werden.

Durch den verhältnismäßig hohen Prozentsatz von Analphabeten in Papua und Neuguinea (teils über 90\% Volksbildung eine besondere Aufgabe zu. Deswegen forderte die Internationale Bank für Aufbau und Entwicklung, New York (UNO), in einem Bericht zur wirtschaftlichen Lage und Entwicklung des Territoriums vom Jahre 1964 die Errichtung von privaten Sendern. Den bestehenden oder geplanten Sendern der Regierung oder halbamtlicher Stellen ( $A B C)$ wird dabei eine ergänzende Rolle zugewiesen. In dem auf Wunsch der australischen Regierung erstellten umfangreichen Bericht heißt es dazu u. a.: „... the Mission considers it important, that the possibility of reaching the indigenes at the local level should not be restricted to official programs. As the sole means of widespread communication among an almost illiterate adult population, radio is clearly of primary importance as a means of conveying information and of forming public opinion. As such, free radio is of the same importance as a free press in literate countries. It cannot be denied that problems will result if diverse and perhaps conflicting statements and ideas are put out by stations, operated by different interests. The way to solve these however, is not by control of programs... This Mission supports in principle the establishment of a network of radio stations capable of giving better coverage than can be obtained with the present installations. It considers, that the $\mathrm{ABC}$ and the Administration have complementary roles to play in the production of broadcast programs ... ${ }^{44}$

$\mathrm{Da}$ in Australien selbst schon seit Beginn des Rundfunks auch kommerzielle Rundfunkstationen bestehen, ist zu erwarten, daß in absehbarer Zeit auch in Papua und Neuguinea privaten Trägern (etwa den christlichen Missionen) die Möglichkeit gegeben wird, eigene Sender zu errichten. Die Überlegungen des Broadcasting Advisory Council von Papua und Neuguinea über die Errichtung kommerzieller Stationen oder die Zulassung nichtkommerzieller Stationen, die von den verschiedenen christ- 
lichen Missionen des Landes betrieben werden sollen, waren um die Jahreswende 1965/1966 noch nicht abgeschlossen.22

Einen eigenen "Uberseesender" für das Territorium forderte John Guise in seiner Jungfernrede als frischgewählter Leiter der Gruppe der einheimischen Abgeordneten im House of Assembly, Port Moresby, im August 1964. Diese Station solle nach dem Muster von Radio Australia arbeiten, das überall gut aufgenommen werde und viel Lob für den hohen Standard seines Programms erhalte. Es sei wesentlich, so meinte Guise, Parlamentarischer Unterstaatssekretär für Information and Extension Services, daß Nachrichten und allgemeine Berichte über Papua und Neuguinea verbreitet würden. Gerade ein solcher Sender würde der Sache der Bewohner des Territoriums im Kampf der Meinungen nur helfen. ${ }^{25}$

Bezüglich des Fernsebens, das bisher im Territorium noch nicht existiert, sieht die Fach-Delegation der Bank für Aufbau und Entwicklung, New York, keinen Grund, es nicht auch in Papua und Neuguinea einzuführen. So wie in Fragen des Verkehrs bereits seit langem die modernsten Mittel, nämlich Flugzeuge, eingesetzt würden, heißt es in dem Gutachten, sei kein Grund einzusehen, weshalb nicht auch für Erziehung und Bildung der Bevölkerung das modernste Medium, eben das Fernsehen benutzt werden solle. Deswegen empfiehlt die Bank: „The Mission recommends that a technical survey of the television potential should be made and that, if the results in any way justify it, one or more pilot projects be started. " 28 Tatsächlich hat das House of Assembly in Port Moresby in seiner Januar-Sitzung 1965 über die Errichtung eines Fernsehnetzes für Erziehungs- und Bildungsaufgaben gesprochen und einen entsprechenden Ausschuß eingesetzt. ${ }^{27}$

\section{Anmerkungen:}

20. Die ersten Postagenturen in Deutsch-Neuguinea wurden 1887, drei Jahre nach der Ubernahme durch die Neuguinea Compagnie, errichtet. Vgl. Lathe, Ursprung und Entwicklung des Post- und Telegraphenwesens der deutschen Schutzgebiete in der Südsee und in China, in: Karl Schneider (Hrsg.), Jahrbuch über die deutschen Kolonien, Essen, VI: 1913, $143 \mathrm{ff}$.

21. Vgl. R. W. Robson / J. Tudor (Hrsg.), Handbook of Papua and New Guinea, Sydney 1964, 18. Ian K. Mackay, Administration Broadcasting in Papua and New Guinea I, in: "Australian Territories“, Canberra, 7:1967, 13 (January--June), 29.

22. Vgl. J. A. Gibson, Radio and its functions in Australian Territories in: „Australian Territories", Canberra, V: 1965, No. 2, 17-23.

23. Gegen Ende 1965 hat Radio Wewak seine Sendezeit so erweitern können, daß die Sendungen samstags und sonntags bereits um $13.15 \mathrm{Uhr}$ beginnen. An anderen Tagen beginnen die Sendungen jetzt um 16.15 Uhr. Alle Programme schließen um 22.30 Uhr.

24. The Economic Development of the Territory of Papua and New Guinea, Report of a Mission Organized by the International Bank for Reconstruction and Development, Baltimore/Maryland 1965, 326.

25. Vgl. „South Pacific Post ${ }^{\alpha}$, Port Moresby, 4. Sept. 1964, 12

26. Vgl. Anm. 24!

27. Nach einer Notiz in „Pacific Islands Monthly" bildeten Herbst 1965 Prof. Derek Broadbent und Douglas Brooke ein Untersuchungsgremium zu Fragen des Fernsehens im Territorium. Seine Aufgabe ist es, "to inquire into... the usefulness, technical feasibility and costs of television in the Territory of Papua and New Guinea, not only as an educational medium but as a medium for mass communication and entertainment". („Pacific Islands Monthly“, Sydney, 37: 1966, No. 1,20) - Der inzwischen erstellte und veröffentlichte Vorschlag ist so großzügig und kostspielig, daß er kaum Aussicht auf Verwirklichung hat. 
As far as Radio is concerned, there are two different types of stations. Beside two Radio stations belonging to the Australian Broadcasting Commission (ABC) - located in Port Moresby and Rabaul - there are local radio stations operated by the administration of the Territory. These stations are especially designed to the needs of the native people. They use Pidgin-English or Police-Motu and local languages. Six such stations are already operating others, in nearly all the main places of the Territory, are in the stage of planning or construction.

\title{
R ESUMEN I I
}

En lo que se refiere a la Radio, hay dos clases de estaciones. Además de dos estaciones de Radio pertenecientes a la Australian Broadcasting Commission (ABC) - ubicadas en Port Moresby y Rabaul - existen estaciones de Radio locales dirigidas por la administración del Territorio. Estas estaciones se destinan especialmente para salir al encuentro de las necesidades de los nativos. Usan Pidgin-English o Police-Motu y otras lenguas locales. $\mathrm{Ya}$ operan seis de estas estaciones, estando otras, en casi todos los centros más importantes del Territorio, en estadio de planificación o construcción.

\section{Probleme und Möglichkeiten der katholischen Presse in Afrika}

\author{
von Al Imfeld
}

Die christlichen Missionen im afrikanischen Kontinent haben Pionierarbeit auf dem Gebiet der Publizistik geleistet. Studien, die der Geschichte des geschriebenen und gedruckten Wortes in Afrika südlich der Sahara oder im sogenannten "Schwarzen Afrika" (und darum geht es in diesem Artikel) nachgehen, stehen vor der Tatsache, daß die christliche Publizistik ,und zwar vor allem ihr katholischer Zweig - wohl die älteste gedruckte Publizistik im Schwarzen Kontinent ${ }^{\text {" }}$ ist. $^{1}$

In seinem Afrikabuch stellte Johannes Beckmann 1947 fest, daß in verhältnismäßig kurzer Zeit rein quantitativ überraschend viel geleistet wurde. ${ }^{2}$ Schon Ende des 19. Jahrhunderts gab es zahlreiche Missionsblätter in Afrika. Die Verdienste der Missionare sind unbestritten. Sie haben das Alphabet in den Busch und später in die Stadt gebracht. Sie lehrten die Afrikaner lesen und nachher waren sie es auch, die durch ihre Missionsblätter den Bekehrten Lesestoff zur Weiterbildung boten.

Die „Bibliotheca Missionum“ verzeichnet für einen Zeitraum von 30 Jahren, zwischen 1910 und 1940, 342 Titel von katholischen Zeitungen und Zeitschriften. ${ }^{3}$ Nikolaus

Al Imfeld MA, MSJ ist in den USA ausgebildeter Kommunikationswissenschaftler. Er arbeitet z. Z. in Fort Victoria (Rhodesien) an Untersuchungen über die afrikanische Publizistik. Uber den hier veröffentlichten Aufsatz hinaus hat Imfeld uns eine von ihm erstellte Bibliographic zur Verfügung gestellt, die im Vorfeld der Publizistik dem Studium afrikanischer Probleme dienen kann und dazu anregen soll. Interessenten können sich wegen dieser Bibliographie an die römische Redaktion von CS wenden. 